\title{
Modeling of working environment and coverage path planning method of combine harvesters
}

\author{
En $\mathrm{Lu}^{1,2}$, Lizhang $\mathrm{Xu}^{1 *}$, Yaoming $\mathrm{Li}^{1}$, Zhong Tang ${ }^{1}$, Zheng $\mathrm{Ma}^{1}$ \\ (1. School of Agricultural Equipment Engineering, Jiangsu University, Zhenjiang 212013, China; \\ 2. World Precise Machinery (China) Co., Ltd, World Industrial Park, Picheng Town, Danyang 212311, China)
}

\begin{abstract}
This paper mainly studied the working environment modeling and coverage path planning of combine harvesters. The boundaries of the farmland to be harvested were extracted through the farmland satellite imagery and canny algorithm. The polygon approximation method was used to fit the extracted boundaries as polygons. The edge offset of the farmland and obstacles was realized based on the principle of straight skeleton. According to the structure data of the split points which were obtained through the improved scan line algorithm, the coverage path planning of the combine harvester was realized. Moreover, the circular arc transition algorithm was used to optimize the harvesting paths to achieve the smooth turning of the combine harvester at the edge of farmland and when encountering obstacles. The simulation results show that the proposed the proposed polygon approximation method can accurately depict the boundaries of the farmland to be harvested, and reduce the amount of data to be stored. Additionally, the designed path planning method can realize the coverage path planning of the combine harvester in irregular and internal obstacle farmland.
\end{abstract}

Keywords: combine harvester, environmental modeling, path planning, farmland turning

DOI: $10.25165 /$ j.ijabe.20201302.5210

Citation: Lu E, Xu L Z, Li Y M, Tang Z, Ma Z. Modeling of working environment and coverage path planning method of combine harvesters. Int J Agric \& Biol Eng, 2020; 13(2): 132-137.

\section{Introduction}

With the development and improvement of navigation and positioning technology, it is gradually being used in agricultural machinery to achieve unattended operation ${ }^{[1]}$. This way can improve the automation of agricultural machinery and reduce labor intensity under some specific circumstances (such as traveling straight $)^{[2]}$. Because the boundaries of farmland are complex and there are often many obstacles inside, the actual working environments of combine harvesters are often very harsh. At this time, the combine harvesters with auxiliary navigation systems require switch to the mode of manual driving at the edge of farmland and when encountering obstacles. In this way, only a small part of the harvesting paths can achieve straight automatic harvesting, and the actual work effect is obviously poor. This is mainly due to technical defects in the working environment modeling and path planning of the combine harvester. Therefore, it is important to study the accurate and efficient environment modeling method for the farmland, and realize the path planning of combine harvesters on the basis of this ${ }^{[3]}$, which is of great significance for realizing the automatic harvesting of combine harvesters.

Received date: 2019-06-18 Accepted date: 2019-11-15

Biographies: En Lu, PhD, Assistant researcher, research interests: intelligent agricultural robotics, complex system modeling, and nonlinear control, Email: jsluen@163.com; Yaoming Li, PhD, Professor, research interests: intelligent agricultural machinery, Email: ymli@ujs.edu.cn; Zhong Tang, PhD, Associate researcher, research interests: intelligent agricultural machinery, Email: zht@ujs.edu.cn; Zheng Ma, PhD, Associate researcher, research interests: intelligent agricultural machinery, Email: mazheng123@ujs.edu.cn.

*Corresponding author: Lizhang $\mathrm{Xu}, \mathrm{PhD}$, Researcher, research interests: intelligent agricultural machinery, complex system modeling. School of Agricultural Equipment Engineering, Jiangsu University, Zhenjiang 212013, China. Tel: +86-13815171180, Email: justxlz@ujs.edu.cn.
Establishing the environmental model of the farmland to be harvested is the prerequisite of path planning of the combine harvester ${ }^{[4]}$. Jensen et al. ${ }^{[5]}$ used the Geographic Information System (GIS) to obtain the road network information, and combined with the farmland mapping information, the environment model of the working area was established. Korkmaz et al. ${ }^{[6]}$ proposed an environment modeling method for the disaster rescue vehicle based on the segmented satellite imagery. This method requires a large amount of data to be stored. Zhao et al. ${ }^{[7]}$ used the Gauss-Kruger projection formula to convert the GPS coordinates of the four vertices of the crab pond into plane coordinates, and then the working area coordinate system of the crab pond was constructed. This method can only be used for the working area environment modeling with regular shapes. Liu et al. ${ }^{[8]}$ measured the topography of the farmland through the Global Navigation Satellite System (GNSS) installed on the tractor. Based on this, the environment model of the farmland was established. This method is not well suited for the environment modeling of working area with complex boundaries and many obstacles.

Related scholars are continually working to develop the coverage path planning for the robotic automation control ${ }^{[9]}$. Oksanen et al. ${ }^{[10]}$ used the trapezoidal decomposition algorithm to decompose complex farmland model into multiple sub-blocks, and carried out path planning research on the sub-blocks. Its computational efficiency is greatly dependent on the complexity of the farmland model. Zhou et al. ${ }^{[11]}$ divided the farmland model into sub-blocks according to the distribution of obstacles, and the ant colony algorithm was used to optimize the harvesting order of the sub-blocks. Zhu et al. ${ }^{[12]}$ studied the coverage path planning of underwater autonomous vehicles based on the Glasius Bio-inspired Neural Network (GBNN) algorithm, and optimized the paths at the edge of obstacles through some typical path templates. This method requires the use of raster maps to define biological neurons, so it requires a large storage space to store 
model data and has a high computational complexity. Based on the element decomposition method and ant colony algorithm, Zhang et al. ${ }^{[13]}$ proposed a coverage path planning method combining local region traversal and global motion planning. However, the ant colony algorithm may fall into local optimum when solving, and there is no guarantee that the optimized path is optimal. Tang et al. ${ }^{[14]}$ used the rectangular decomposition method to divide the working environment model into several sub-areas, and combined the adjacency matrix and path selection function to realize the coverage path planning of the wall-climbing robot. It also has the problem of data redundancy.

In order to facilitate mechanization, the boundaries of modern farmland are mostly polygonal. Therefore, this paper considers the use of polygons to describe the boundaries of farmland, and the harvesting paths of the combine harvester are planned based on this. Firstly, the satellite maps are used to obtain the high-resolution images of farmland. After that, the boundaries of the grayscale farmland image are identified by the canny algorithm, and fitting them into polygons by the polygon approximation method. Subsequently, the coverage path planning of the combine harvester is realized by the improved scan line algorithm and the circular arc transition algorithm. Finally, the simulation results are given to show the effectiveness of the proposed working environment modeling and coverage path planning method.

This study aims to investigate the working environment modeling method of farmland and the path planning strategy of combine harvesters. The paper is organized as follows: The working environment modeling method of farmland is explained in the following section. The subsequent section gives the key division point acquisition method. Then, the coverage path planning of the combine harvester is implemented next. The final section presents a brief summary of the study.

\section{Environment modeling}

The satellite image of the farm obtained through Google Maps is shown in Figure 1.

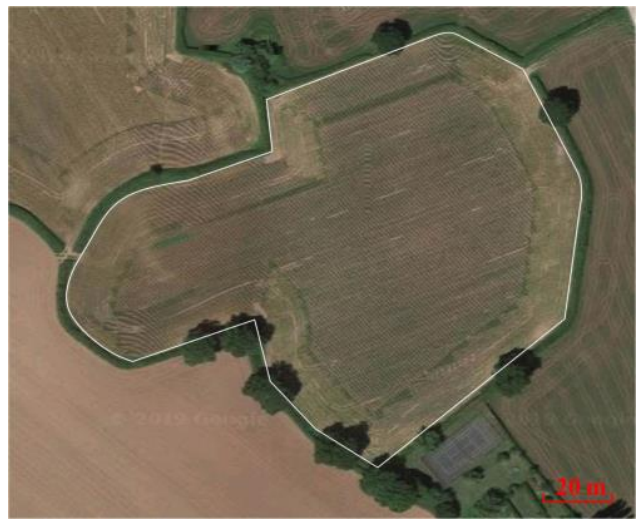

Figure 1 Satellite image of the farmland

The grayscale image processing method is used to process the satellite image of the farmland ${ }^{[15]}$, as shown in Figure 2a. The canny algorithm is an edge detection operator that uses a multi-stage algorithm to detect a wide range of edges in images. It can be seen from Figure 2a, the grayscale value of the farmland grayscale image has a significant change, so the grayscale value can be used to extract the boundaries of the farmland based on the canny algorithm ${ }^{[16]}$. Moreover, the boundaries of farmland or obstacles are always closed. Therefore, we have adopted a closed curve detection method to find the closed curve in the extracted multiple boundaries information which is obtained by the canny algorithm, so that the real farmland boundary information can be found, and the wrong boundary information (such as noise) can be filtered out. Finally, the extracted farmland boundaries expressed in pixels are shown in Figure $2 b$.

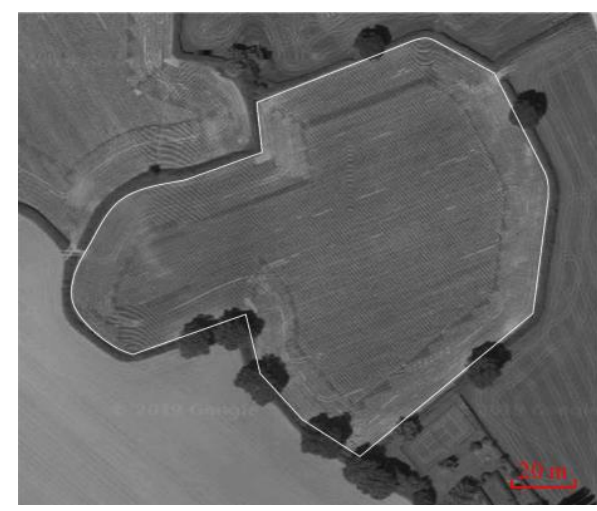

a. Farmland grayscale image

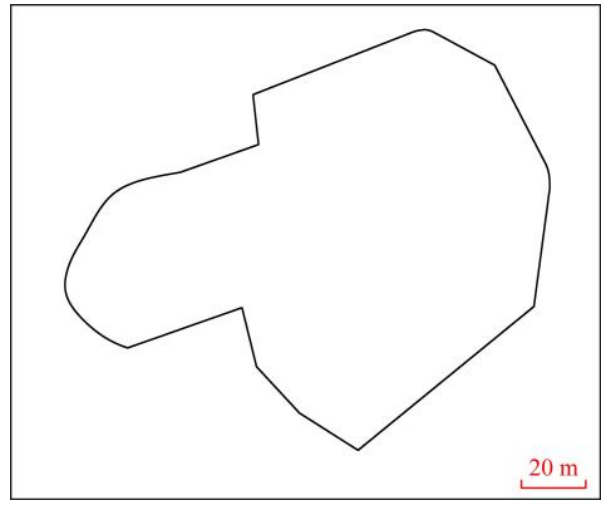

b. Farmland boundaries

Figure 2 Grayscale processing and boundary extraction of the farmland

The boundaries of the farmland can be expressed as $b=\left\{p_{1}\right.$, $\left.p_{2}, \ldots \ldots p_{n}\right\}$, where $p_{i}$ is the coordinates of the $i_{t h}$ pixel in the $b$.

A straight line passing through two pixels $p_{a}\left(x_{a}, y_{a}\right)$ and $p_{b}\left(x_{b}\right.$, $y_{b}$ ) can be expressed as ${ }^{[17]}$ :

$$
x_{0}\left(y_{a}-y_{b}\right)+y_{0}\left(x_{b}-x_{a}\right)+y_{b} x_{a}-y_{a} x_{b}=0
$$

where, $x_{0}$ and $y_{0}$ represent the horizontal and vertical coordinates on the straight line passing through the two pixels $p_{a}\left(x_{a}, y_{a}\right)$ and $p_{b}\left(x_{b}, y_{b}\right)$.

The deviation $d_{i}$ of the $i_{t h}$ pixel $p_{i}\left(x_{i}, y_{i}\right)$ and the straight line passing through the two pixels $\left\{p_{1}, p_{n}\right\}$ in the boundaries $b$ of the farmland can be expressed as ${ }^{[17]}$ :

$$
d_{i}=\left|x_{i}\left(y_{1}-y_{n}\right)+y_{i}\left(x_{n}-x_{1}\right)+y_{n} x_{1}-y_{1} x_{n}\right|
$$

Assuming that the maximum deviation pixel is $p_{\max }$, the pixel pairs can be written as $\left\{p_{1}, p_{\max }\right\}$ and $\left\{p_{\max }, p_{n}\right\}$. Therefore, two new pixel intervals can be found from the boundaries $b$ of the farmland using the Equations (1) and (2). On this basis, repeat the above process until all linear deviations meet the maximum deviation conditions:

$$
\max \left(d_{i}\right)<d_{t h}
$$

where, $d_{t h}$ is the selected threshold.

Finally, the boundaries of the farmland to be harvested can be approximated as a polygon, as shown in Figure 3. In the Figure 3a, the blue solid lines are the boundaries of the farmland, and the red dotted lines are the boundaries of the farmland after the polygon approximated. It can be seen that the result of the polygonal approximation is in good agreement with the boundaries of the farmland, and the maximum approximation error of each side of the polygon is shown in Figure 3b. This shows that the 
proposed farmland environment modeling method can effectively realize the working environment modeling of the combine harvester.

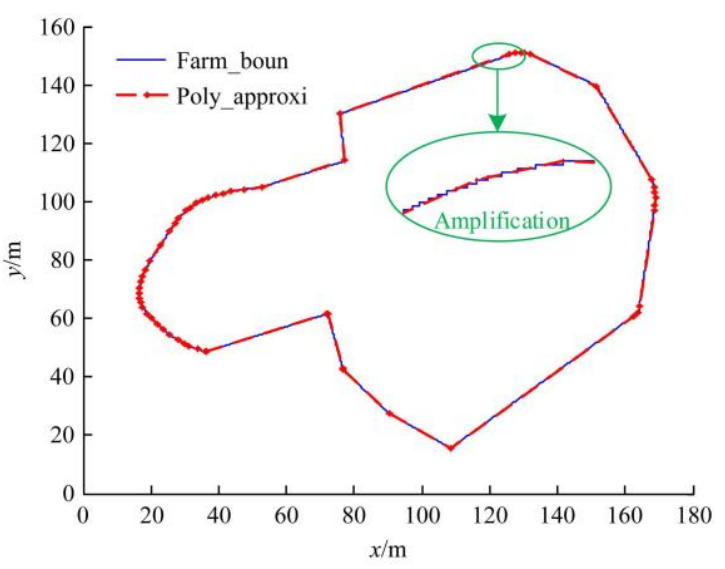

a. Polygon approximation

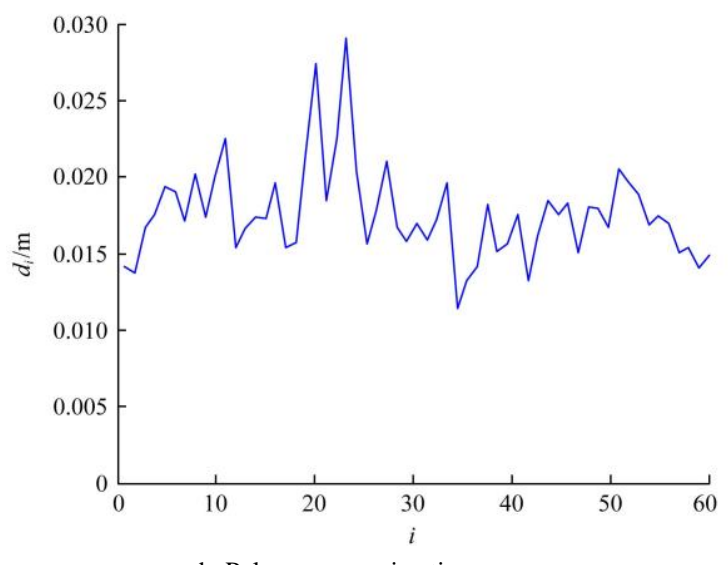

b. Polygon approximation errors

Figure 3 Polygon approximation of the farmland

\section{Planning pretreatment}

\subsection{Edge offset algorithm based on straight skeleton principle}

In the previous section, the farmland to be harvested described in pixels is converted into data described by the polygons. It is necessary to ensure safety first when the combine harvester is performing automatic harvesting operations. The solution is to first perform an edge offset on the established farmland environment model before the coverage path planning of the combine harvester. According to the type of polygon vertices, the straight skeleton of the polygons can be solved. The offset line segments are obtained by the monotonous small areas formed by the straight skeleton. Finally, the obtained offset line segments are connected into a closed offset polygon. The flow of the algorithm is shown in Figure $4^{[18]}$.

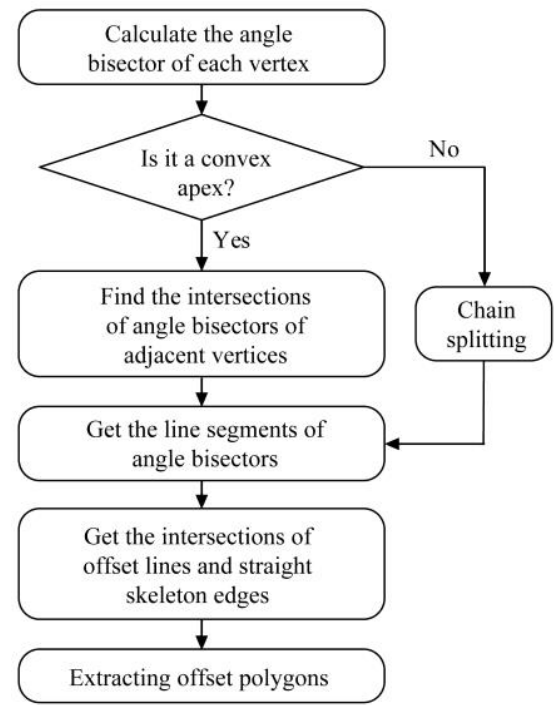

Figure 4 Flow chart of contour offset algorithm based on the straight skeleton principle

The common obstacles in farmland are telegraph poles, trees, ponds, mounds, etc., as shown in Figure 5. The shapes of the obstacles are usually circular and polygonal in the actual field conditions. Therefore, in order to reflect the generality of the algorithm, a circular and a quadrilateral obstacle are added to the farmland to be harvested. Subsequently, the edge offset on the established farmland environment model is performed using the contour offset algorithm based on the straight skeleton principle, and as shown in Figure 6. Where the blue lines are the boundaries of the farmland, and the red dotted lines are the boundaries after the edge offset.
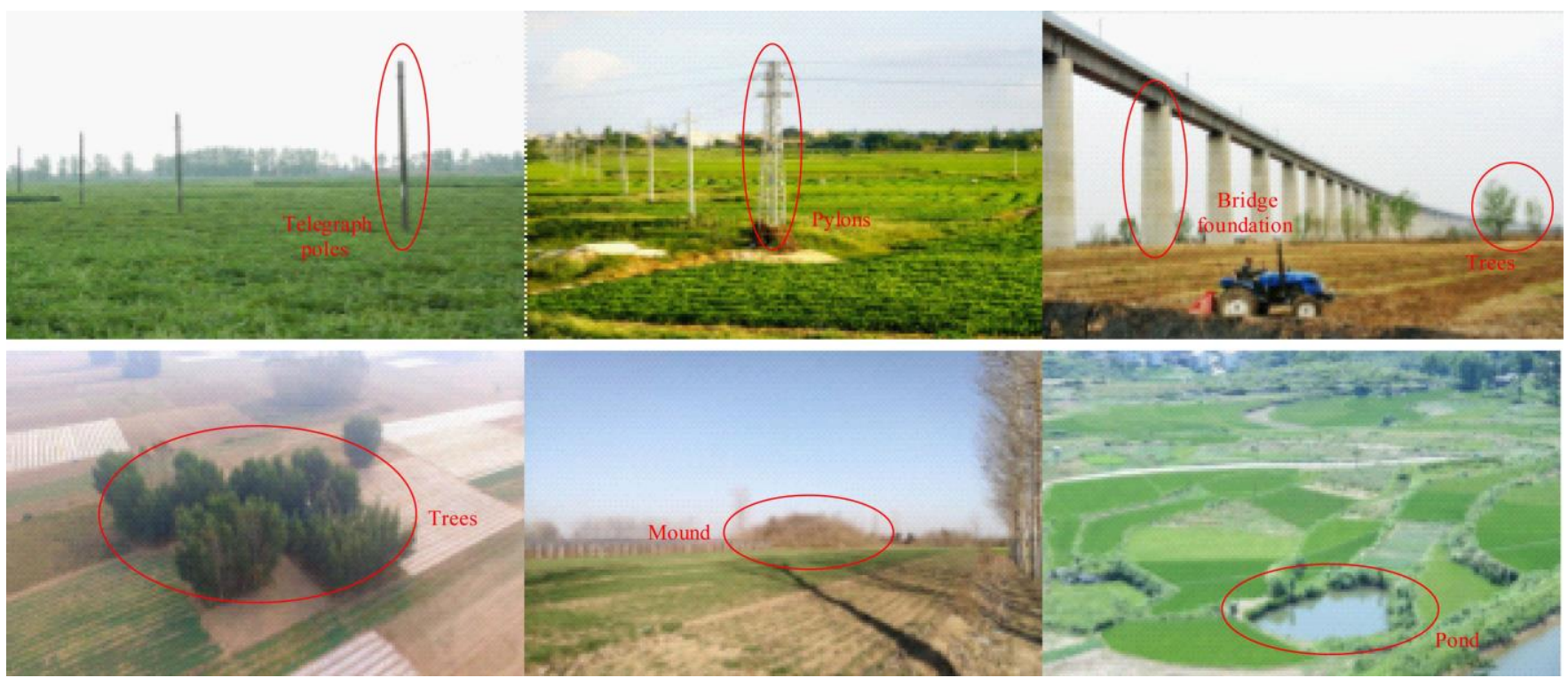

Figure 5 Common obstacles in farmland 


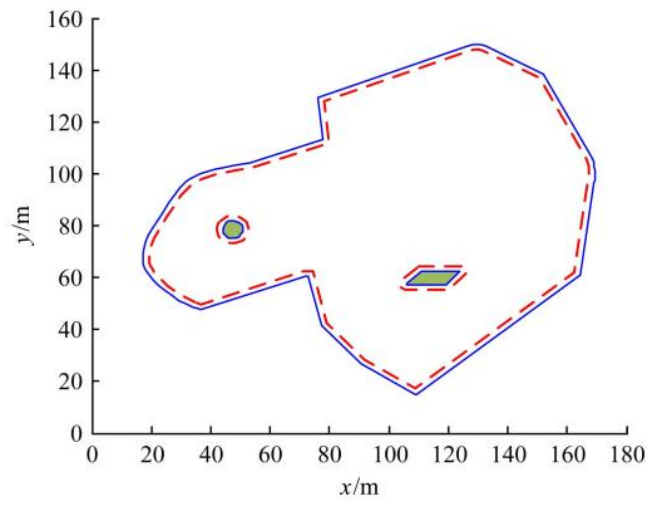

Figure 6 Farmland to be harvested after edge offset

\subsection{Improved scan line algorithm}

As shown in Figure 7, the farmland to be harvested is in the $x y$ coordinate system. Therefore, the farmland to be harvested can be moved to the $n p$ coordinate system by coordinate transformation, wherein the $p$ coordinate is parallel to the scan line, and the coordinate transformation is as shown in Equation (4) ${ }^{[19]}$.

$$
\left[\begin{array}{l}
n \\
p
\end{array}\right]=\frac{1}{d}\left[\begin{array}{ll}
n_{x} & n_{y} \\
p_{x} & p_{y}
\end{array}\right]\left[\begin{array}{l}
x \\
y
\end{array}\right]
$$

where, $d$ is the harvester table width; $n_{x}, n_{y}$ is the coordinate of the unit vector $n$ in the $x y$ coordinate; $p_{x}, p_{y}$ is the coordinate of the unit vector $p$ in the $x y$ coordinate.

The inverse coordinate transformation is as shown in Equation (5):

$$
\left[\begin{array}{l}
x \\
y
\end{array}\right]=d\left[\begin{array}{ll}
n_{x} & n_{y} \\
p_{x} & p_{y}
\end{array}\right]^{-1}\left[\begin{array}{l}
n \\
p
\end{array}\right]
$$

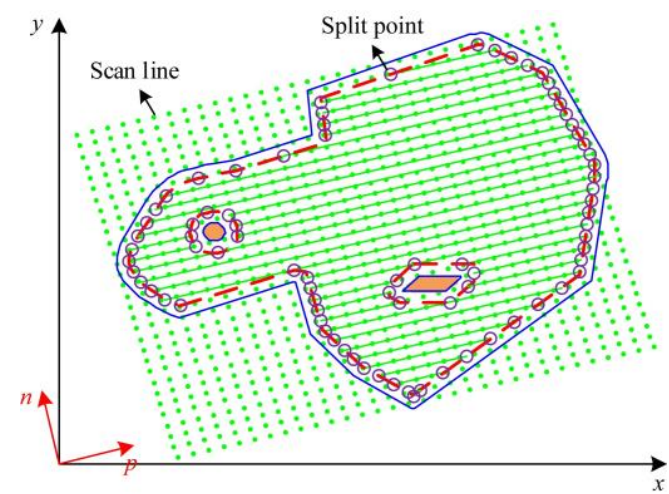

Figure 7 Schematic diagram of the scan line algorithm

The intersection points of the scan line and the polygons (environment model) is called the split points, and as shown by the purple circles in the Figure 7. The line segment composed of split points $i, i+1$ is defined as $i$, and its slope coefficient at $n p$ coordinates can be expressed as:

$$
k=\frac{p_{i+1}-p_{i}}{n_{i+1}-n_{i}}
$$

Then,

$$
p(0)=p_{i}-k n_{i}
$$

The other $p$ coordinates corresponding to the $n$ coordinate can be expressed as:

$$
p(i)=k m+p(0), \quad m=\left\lceil\min _{k=1,2} n_{k}\right\rceil, \cdots \cdots,\left\lfloor\max _{k=1,2} n_{k}\right\rfloor
$$

Combined with Equations (6), (7) and (8), you can get:

$$
p(i)=\frac{p_{i+1}-p_{i}}{n_{i+1}-n_{i}}\left(m-n_{i}\right)+p_{i}, \quad m=\left\lceil\min _{k=1,2} n_{k}\right\rceil, \cdots . .\left\lfloor\max _{k=1,2} n_{k}\right\rfloor
$$

The structure is used to record the information about the split points $^{[20]}$, and shown as follows:

\section{struct Struct_Node \{}

Point : Split point coordinates;

Type : Split point type;

Visited : Mark whether the split point has been traversed;

Segment : Mark edge;

Polygon : Mark polygon;

\}

\section{Coverage path planning}

According to the structure data of the split points, the preliminary coverage path planning is performed, and the flow chart is shown in Figure 8. The results of the preliminary coverage path planning are shown in Figure 9. In the Figure 9, the green solid lines are the traveling paths of the combine harvester during harvesting, and the black dotted lines are the traveling paths in which the combine harvester does not perform the harvesting operation.

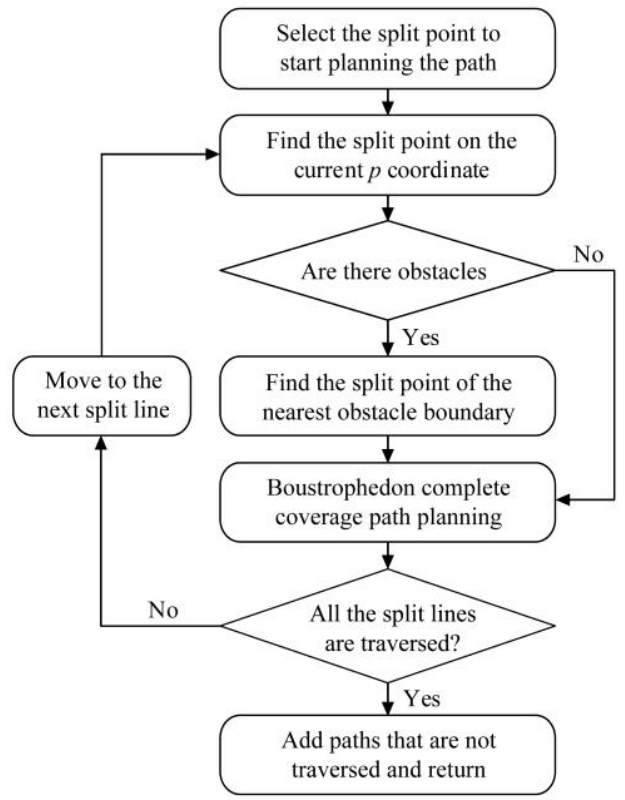

Figure 8 Flow chart of preliminary coverage path planning

When the combine harvester reaches the edge of farmland, it needs to realize its automatic turn-row, and when encountering obstacles, it also needs to turning around. It can be seen from Figure 9 that the preliminary coverage path planning does not consider the combine harvester's turning at the edge of farmland and when encountering obstacles. Therefore, it is necessary to study the turning mode and path generation algorithm of the combine harvester ${ }^{[21]}$. This paper proposes a circular arc transition algorithm to achieve the smooth turning of the combine harvester. As shown in Figure 10, let the two endpoints of a line be $q_{1}\left(x_{1}, y_{1}\right)$ and $q_{2}\left(x_{2}, y_{2}\right)$, the center of the circle is $c\left(x_{c}, y_{c}\right)$, and the radius is $r$, then if there is an intersection point between the line and the circle $q(x, y)$, you can get:

$$
\overrightarrow{o q}=\overrightarrow{o q_{1}}+u\left(\overrightarrow{o q_{2}}-\overrightarrow{o q_{1}}\right)
$$

where, $0 \leq u \leq 1$. Equation (10) can be converted into the following form:

$$
\left\{\begin{array}{l}
x=x_{1}+u\left(x_{2}-x_{1}\right) \\
y=y_{1}+u\left(y_{2}-y_{1}\right)
\end{array}\right.
$$

Since the intersection point $q$ is also on the circle, then,

$$
\left(x-x_{c}\right)^{2}+\left(y-y_{c}\right)^{2}=r^{2}
$$


Combined with Equations (10), (11) and (12), you can get:

$$
A u^{2}+B u+C=0
$$

where, $\quad A=\left(x_{2}-x_{1}\right)^{2}+\left(y_{2}-y_{1}\right)^{2} \quad, \quad B=2\left(\left(x_{2}-x_{1}\right)\left(x_{1}-x_{c}\right)+\right.$ $\left.\left(y_{2}-y_{1}\right)\left(y_{1}-y_{c}\right)\right), \quad C=x_{c}^{2}+y_{c}^{2}+x_{1}^{2}+y_{1}^{2}-2\left(x_{c} x_{1}+y_{c} y_{1}\right)-r^{2}$.

Solving the quadratic equation shown in Equation (13) can be obtained:

$$
u=\frac{-B \pm \sqrt{B^{2}-4 A C}}{2 A}
$$

According to the $B^{2}-4 A C$, the number of intersections between the line and circle can be judged. If $B^{2}-4 A C$ is less than 0 , it means there is no intersection; if $B^{2}-4 A C$ is equal to 0 , it means tangency and only one intersection; if $B^{2}-4 A C$ is greater than 0 , it means there are two intersections. Based on this, the circular arc transition of two line segments can be achieved.

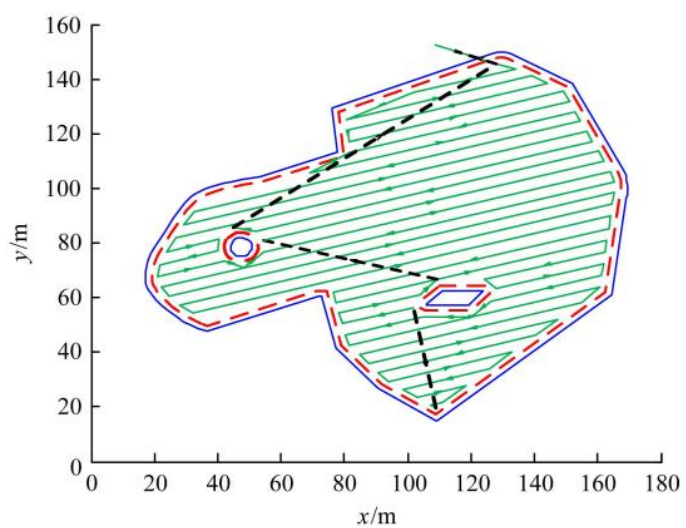

Figure 9 Preliminary coverage path planning

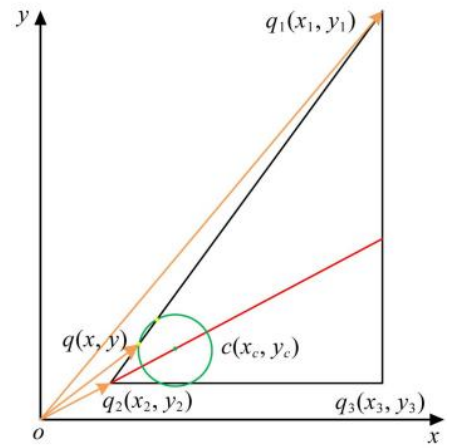

Figure 10 Circular arc transition algorithm

Combined with the circular arc transition algorithm, the harvesting area is re-planned, and the coverage path planning considering turning mode is shown in Figure 11. It can be seen from Figure 11 that the circular arc transition of the combine harvester is realized at the edge of farmland and when encountering obstacles.

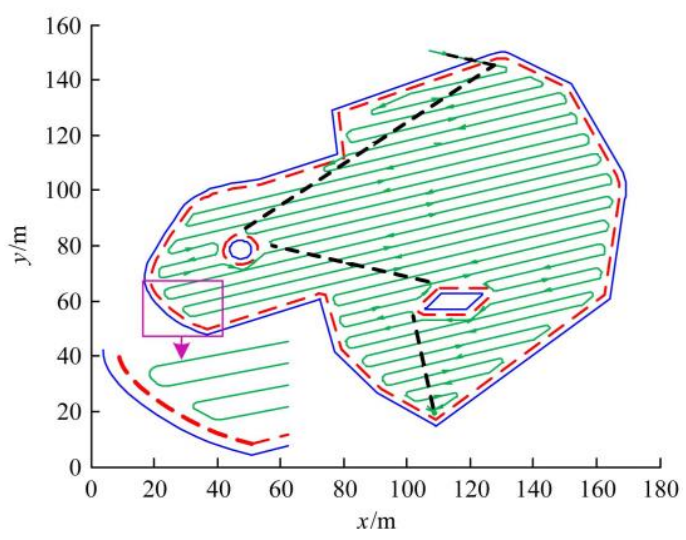

Figure 11 Coverage path planning considering turning mode

\section{Conclusions}

In this study, a working environment modeling and coverage path planning method of combine harvesters is presented. The polygonal approximation method is applied to fit the boundaries of farmland. Combining edge offset and improved scan line algorithm, the preliminary coverage path planning of the combine harvester is implemented. Subsequently, the circular arc transition algorithm is used to optimize the harvesting paths to ensure that the combine harvester's turning at the edge of the farmland and when encountering obstacles, and the numerical simulations are presented.

It can be seen from the results of environmental modeling that the proposed polygon approximation method can accurately depict the boundaries of the farmland to be harvested. It reduces the amount of data that needs to be stored, and reduces the difficulty of the combine harvester path planning. The simulation results of path planning show that the proposed method can realize the coverage path planning of the combine harvester. And the traps in path planning can be automatically jumped out in the presence of obstacles. The above results show that the method proposed in this paper can effectively establish environmental modeling of farmland and realize the coverage path planning of combine harvesters, providing the basis for the combine harvester autonomous harvesting.

\section{Acknowledgments}

This research was supported by the earmarked fund for China Agriculture Research System CARS-12, China Postdoctoral Science Foundation Grant (2019M651962), Jiangsu Agriculture Science and Technology Innovation Fund (JASTIF) CX(19)3083, Jiangsu Province "Six Talent Peaks" innovative talent team (TD-GDZB-005), Key R\&D plan of Zhenjiang industry prospect and common key technology (GZ2017001), and A Project Funded by the Priority Academic Program Development of Jiangsu Higher Education Institutions (No. PAPD-2018-87).

\section{[References]}

[1] Yin $\mathrm{X}$, Du J, Noguchi N, Yang T X, Jin C Q. Development of autonomous navigation system for rice transplanter. Int J Agric \& Biol Eng, 2018; 11(6): 89-94.

[2] Luo C M, Li W, Fan X N, Yang H, Ni J J, Zhang X W, et al. Positioning technology of mobile vehicle using self-repairing heterogeneous sensor networks. Journal of Network and Computer Applications, 2017; 93, 110-122.

[3] Dou W H, Zhu K, Liang S H, Wen W S, Guo Y, Tan Y. Path planning algorithm of field robot based on topological map and robot control. China Sciencepaper, 2016; 11(22): 2525-2530.

[4] Rahman M M, Ishii K, Noguchi N. Optimum harvesting area of convex and concave polygon field for path planning of robot combine harvester. Intelligent Service Robotics, 2019; 12(2): 167-179.

[5] Jensen M A F, Bochtis D, Sørensen C G, Blas M R, Lykkegaard K L. In-field and inter-field path planning for agricultural transport units. Computers \& Industrial Engineering, 2012; 63(4): 1054-1061.

[6] Korkmaz S A, Poyraz M. Path planning for rescue vehicles via segmented satellite disaster images and GPS road map. International Congress on Image and Signal Processing, BioMedical Engineering and Informatics (CISP-BMEI), 2017.

[7] Zhao D A, Luo J, Sun Y P, Hong J Q, Zhang J. Design and experiment of navigation control system of automatic operation boat in river crab culture. Transactions of the CSAE, 2016; 32(11): 181-188. (in Chinese)

[8] Liu G, Kang X, Xia Y X, Jing Y P. Global path planning algorithm and experiment based on GNSS-controlled precise land leveling system. Transactions of the CSAM, 2018; 49(5): 27-33. (in Chinese) 
[9] Patle B K, Ganesh B L, Pandey A, Parhi D R K, Jagadeesh A. A review: On path planning strategies for navigation of mobile robot. Defence Technology, 2019; 15(4): 582-606.

[10] Oksanen T, Visala A. Path planning algorithms for agricultural machines. Journal of Field Robotics, 2010; 26(8): 651-668

[11] Zhou K, Leck Jensen A, Sørensen C G, Busato P, Bothtis D D. Agricultural operations planning in fields with multiple obstacle areas. Computers and Electronics in Agriculture, 2014; 109: 12-22.

[12] Zhu D Q, Tian C, Sun B, Luo C M. Complete coverage path planning of autonomous underwater vehicle based on GBNN algorithm. Journal of Intelligent \& Robotic Systems, 2018; 94: 237-249.

[13] Zhang C B, Wang X S. Complete coverage path planning based on ant colony algorithm. China Mechanical Engineering, 2008; 19(16): 1945-1949. (in Chinese)

[14] Tang D L, Yuan B, Hu L, Li M Y, Wei Z B. Complete coverage path planning method for oil tank inspection wall climbing robot. Journal of Engineering Design, 2018; 25(3): 253-261. (in Chinese)
[15] Bhattacharya S, Ghrist R, Kumar V. Persistent homology for path planning in uncertain environments. IEEE Transactions on Robotics, 2015; 31(3): 578-590.

[16] Rami A J, Mohammad A J, Hubert R. A novel edge detection algorithm for mobile robot path planning. Journal of Robotics, 2018; 9: 1-12.

[17] Prasad D K, Leung M K H. Polygonal representation of digital curves. Digital Image Processing, InTech, 2012; pp.71-90.

[18] Luo H, Li D C, Xie R D, Cao Y. An algorithm of contour offsetting based on the principle of straight skeleton for rapid prototyping. Journal of Computer-Aided Design \& Computer Graphics, 2011; 23(11): 1908-1914.

[19] Räisänen V. Peltorobotin reititys "siksak"-täytöllä. Tampere University of Technology, 2008.

[20] Saad M, Maher M L. Shared understanding in computer-supported collaborative design. Computer Aided Design, 1996; 28(3): 183-192.

[21] Rahman M M, Ishii K. Heading estimation of robot combine harvesters during turning maneuveres. Sensors, 2018; 18(5): 1390. 